International Journal of Electrical Engineering and Technology (IJEET)

Volume 11, Issue 4, June 2020, pp. 100-107, Article ID: IJEET_11_04_011

Available online at https://iaeme.com/Home/issue/IJEET?Volume $=11 \&$ Issue $=4$

ISSN Print: 0976-6545 and ISSN Online: 0976-6553

DOI: https://doi.org/10.34218/IJEET.11.4.2020.011

(C) IAEME Publication

Scopus Indexed

\title{
ESTIMATION OF MOBILE SPEED USING DOPPLER OVER FADING CHANNELS
}

\author{
V. Jagan Naveen
}

Professor, Department of Electronics and Communication Engineering, GMR Institute of Technology, Rajam, Andhra Pradesh, India

\section{Kannan}

Professor, Department of Electronics and Communication Engineering, GMR Institute of Technology, Rajam, Andhra Pradesh, India

\begin{abstract}
This paper investigates on the mobile speed estimation of broadband wireless communications with severe inter symbol interference duet to large number of fading channel taps. This research is based on the received signals which consists of unknown transmitted data, selective fading channel, unknown frequency coefficients including line-of-sight (LOS) components, and random receiver noise. Inorder to estimate the speed of mobiles with the received signal power Modified normalized auto-covariance method is used. The developed algorithm shows a better results for Rician, Rayleigh and Nakagami channels. The algorithm provides accurate speed estimation even if the signal-to-noise ratio $(S N R)$ is low. Simulation results shows that the new algorithm is suitable for estimating mobile speed relative to a maximum Doppler of $500 \mathrm{~Hz}$.
\end{abstract}

Key words: Rayleigh channel, Rician channel, Nakagami channel, Doppler Frequency, Signal to Noise ratio.

Cite this Article: V. Jagan Naveen and V. Kannan, Estimation of Mobile Speed Using Doppler Over Fading Channels, International Journal of Electrical Engineering and Technology, 11(4), 2020, pp. 100-107.

https://iaeme.com/Home/issue/IJEET?Volume $=11 \&$ Issue $=4$

\section{INTRODUCTION}

Data networks have shown a growing success in the past decades, after the use of fixed Internet networks all over the world .In the present days due to their large expansion, there is a need for wireless access.

In the nineteenth century when Maxwell showed that the transmission of information could be achieved without the need for a wire called Wireless communication. 
A few years later, experimentations such as those of Marconi proved that wireless transmission may be a reality and for rather long distances. Through the twentieth century, great electronic and propagation discoveries and inventions gave way to many wireless transmission systems.

In radio, multiple-input and multiple-output, or MIMO is adopted by the use of multiple antennas at both the transmitting and receiving end in order to improve communication performance. It is considered as one of the smart antenna technology. MIMO technology has attracted attention in wireless communications,

Since the MIMO technology offers significant increases in link range and data throughput without additional transmit power or bandwidth. These benefits of this technology are achieved because of higher spectral efficiency and link reliability or diversity.

\section{CHANNEL MODEL}

A broadband wireless channel is considered with line of sight (LOS) component of the physical channel fading. The frequency selective Rician fading component is given by

$$
g_{c}(t, \tau)=\frac{g(t, \tau)}{\sqrt{1+k}}+\sqrt{\frac{k}{1+k}} h_{L O S}(t) \delta(\tau)
$$

where $\mathrm{k}$ is the Rice factor, $g(t, \tau)$ is wide-sense stationary uncorrelated scattering (WSSUS) Rayleigh fading with normalized unit energy, and the LOS component is with $f_{d}$ the maximum Doppler frequency, $\theta_{0}$ and $\varphi_{0}$ are the angle of arrival and the initial phase, respectively.[1]

$$
h_{L O S}(t)=\exp \left(j 2 \pi f_{d} t \cos \theta_{0}+j \phi_{0}\right)
$$

Let $p_{T}(\tau)$ and $p_{R}(\tau)$ be the unit-energy transmit pulse shaping filter and receive filter, respectively. Then the composite channel impulse response

$$
\begin{gathered}
h(t, \tau)=p_{T}(\tau) \otimes g_{c}(t, \tau) \otimes p_{R}(\tau) \\
+\sqrt{\frac{k}{1+k}} h_{L O S}(n) \sum_{l=-P 1}^{P 2} \sigma_{l} x(n-l)+v(n)
\end{gathered}
$$

can be accurately converted to the following discrete-time fading channel model

where $x(n)$ is the $n$th transmitted symbol, $v(n)$ is additive white Gaussian noise with average power $\sigma^{2}, h_{l}(n)$ is the $l$ th tap fading channel coefficient at time instant $n, L_{1}$ and $L_{2}$ are non-negative integers, $L=L_{1}$

$$
\sigma_{l}=\int_{-\infty}^{\infty} p_{T}(s) p_{R}\left(l T_{s}-s\right) d s
$$

$+L_{2}+1$ is the Rayleigh fading channel length which depends on the transmit filter, and the receive filter, $h_{L O S}(n)=\exp \left(j 2 \pi f_{d} n T_{s} \cos \theta_{0}+j \varphi_{0}\right)$ is the LOS channel coefficient at time instant $n, T_{s}$ is the symbol interval and at the sampled value at $\tau=l T_{s}$ and it is the convolution between the receive and transmit filters, and $\sum_{\mathrm{l}=-\mathrm{P} 1}^{\mathrm{P}} \sigma_{1}^{2}=1, P_{1}$ and $P_{2}$ are non-negative integers which depend on the transmit and receive filters

$$
\mathrm{E}\left\{\mathrm{h}_{l 1}\left(\mathrm{n}_{1}\right) \cdot \mathrm{h}_{l 2}{ }^{*}\left(\mathrm{n}_{2}\right)\right\}=\mathrm{C}_{l 1, l 2} \mathrm{~J}_{0}\left[2 \pi \mathrm{f}_{\mathrm{d}} \mathrm{T}_{\mathrm{s}}\left(\mathrm{n}_{1}-\mathrm{n}_{2}\right)\right]
$$




$$
y(n)=\frac{1}{\sqrt{1+k}} \sum_{l=-L 1}^{L 2} h_{l}(n) x(n-l)
$$

where $\mathrm{E}(\cdot)$ stands for expectation, $\mathrm{C}_{11,12}$ is the inter-tap correlation coefficient , $(\cdot)^{*}$ denotes complex conjugate, $f_{d}$ is the maximum Doppler frequency, $J 0(\cdot)$ is the zero-order Bessel function. It is observed that for normalized Rayleigh fading channel, $\sum_{l=-l 1}^{l 2} c l, l=1$. It is also observed that $C_{l 1, l 2}$ is normally non-zero for $l 1 \neq l 2$,even if the physical channel $g(t, \tau)$ is WSSUS fading.

The following assumptions are made on the wireless transmitters which uses modulations such as M-ary amplitude shift keying (MASK),M-ary quadrature amplitude modulation (MQAM), M-ary and phase shift keying (MPSK) .A zero-mean random variable with zero cross-correlation and unit auto-correlation modulated data symbol $\mathrm{x}(\mathrm{n})$ is considered.[2-3]

\subsection{Doppler Estimation}

For designing mobile speed estimation algorithm, the second-order and fourth order statistics of the received signal $y(n)$ are considered.

The autocorrelation of the received signal is explained by

$$
\mathrm{R}_{\mathrm{yy}}(\mathrm{m}) \triangleq \mathrm{E}\left\{\mathrm{y}(\mathrm{n}+\mathrm{m}) \cdot \mathrm{y}^{*}(\mathrm{n})\right\}
$$

The autocorrelation of the received signal power is specified by

$$
\mathrm{R}_{|\mathrm{y}| 2|\mathrm{y}| 2(\mathrm{~m})} \triangleq \mathrm{E}\left\{|\mathrm{y}(\mathrm{n}+\mathrm{m})|^{2}|\mathrm{y}(\mathrm{n})|^{2}\right\}
$$

The auto-covariance of the received signal power is defined by

$$
\left.\mathrm{V}_{|\mathrm{y}|}\right|^{2} \mathrm{y}||^{2}(\mathrm{~m}) \triangleq \mathrm{E}\left\{\left[|\mathrm{y}(\mathrm{n}+\mathrm{m})|^{2}-\operatorname{Ryy}(0)\right] \times\left[|\mathrm{y}(\mathrm{n})|^{2}-\operatorname{Ryy}(0)\right]\right\}
$$

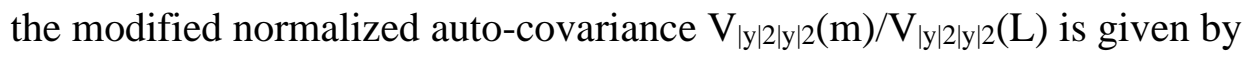

$$
\frac{V_{|y|^{2}|y|^{2}}(m)}{V_{|y|^{2}|y|^{2}}(L)}=\frac{a J_{0}^{2}\left(\omega_{d} m T_{s}\right)+2 k b J_{0}\left(\omega_{d} m T_{s}\right) \cos \left(\omega_{d} m T_{s} \cos \theta_{0}\right)}{a+2 k b}
$$

for $\mathrm{m}>\mathrm{L}$.

where

$$
\begin{gathered}
\boldsymbol{a}=\sum_{l=-L 1}^{L 2} \sum_{s=-L 1}^{L 2}\left|C_{l, s}\right|^{2}, \\
b=\sum_{p=-P_{1}}^{P_{2}} \sum_{\substack{\omega=-P_{1} \\
p=r,}}^{P_{2}} \sum_{\substack{r=-L_{1} \\
\omega=l}}^{L_{2}} \sum_{s=-L_{1}}^{L_{2}} \sigma_{p} \sigma_{\omega}^{*} C_{r, s}
\end{gathered}
$$

Here, $K, a, b$ and $\theta_{0}$ are unknown parameters. Hence, for mobile speed estimation ,autocovariance may not be employed directly[1].

\subsubsection{Second Order Doppler Estimation}

The auto-covariance can be approximated by

$$
\frac{V_{|y|^{2}|y|^{2}}(m)}{V_{|y|^{2}|y|^{2}}(L)} \cong 1-2\left(\pi f_{d} m T_{s}\right)^{2}
$$


, $\mathrm{m}>\mathrm{L}$.

From second order approximation, we can obtain the maximum Doppler estimation as follows:

$$
\hat{f}_{d} \cong \frac{1}{\sqrt{2} \pi m T_{s}} \sqrt{1-\frac{V_{|y|^{2}|y|^{2}}(m)}{V_{|y|^{2}|y|^{2}}(L)}}
$$

\subsubsection{Fourth Order Doppler Approximation}

The maximum Doppler estimation is given by

$$
\begin{aligned}
& \tilde{f}_{d} \cong \frac{1}{\sqrt{3} \pi m T_{s}} \sqrt{2-\sqrt{6 \cdot \frac{V_{|y|^{2}|y|^{2}}}{V_{|y|^{2}|y|^{2}}(L)}-2}} \\
& \mathrm{~m}>\mathrm{L}
\end{aligned}
$$

Through the estimated maximum Doppler, we can calculate the mobile speed via

$v=f_{d} \cdot c / f_{c}$, where $c$ is the speed of light and $f_{c}$ is the carrier frequency and $f_{d}$ is the mean of the second order and fourth order Doppler approximations.

An enhanced practical mobile speed estimation algorithm can be developed using the second order and fourth order Doppler estimations. A single-carrier broadband wireless system is considerd with the transmitted symbols $\{\mathrm{x}(\mathrm{k})\}$. These symbols are partitioned into blocks of length $M$ symbols. Each block is added a cyclic prefix of $\mathrm{M}_{\mathrm{cp}}$ symbols with $\mathrm{M}_{\mathrm{cp}} \geq \mathrm{L}$. The prefix is discarded at the receiver to eliminate inter-block interference.[4-5].

Let $\mathrm{s}_{\mathrm{k}}(n)=\left|y_{k}(n)\right|^{2}$ be the instantaneous power of the $n$th received symbol of the $k$ th block. Pass the symbol $\mathrm{s}_{\mathrm{k}}(\mathrm{n})$ through a low pass filter $\mathrm{f}(\mathrm{l})$ in order to suppress the power fluctuation and noise caused by data symbols, and the filtered power signal is given by $\hat{s}_{k}(n)$ $=\sum_{l=0}^{L f} s_{k}(n-l) . f(l)$, where $L_{f}$ is the order of the low-pass filter.

The auto-covariance of $N$ block filtered signal $\hat{s}_{k}(n)$ is given by

$$
\begin{gathered}
V_{N}^{b}\left(q T_{b}\right)=\frac{1}{M N} \sum_{k=1}^{N-q} \sum_{n=1}^{M}\left[\hat{s}_{k+q}(n)-\bar{s}\right]\left[\hat{s}_{k}(n)-\bar{s}\right], q \geq 1 \\
V_{N}^{s}\left(p T_{s}\right)=\frac{1}{M N} \sum_{k=1}^{N} \sum_{n=1}^{M-p}\left[\hat{s}_{k}(n+p)-\bar{s}\right]\left[\hat{s}_{k}(n)-\bar{s}\right], p \geq L+L_{f}
\end{gathered}
$$

where $T_{b}=\left(M+M_{c p}\right) T_{s}$ is the time duration of a block and $T_{s}$ is the symbol interval. Mean of the signal, is given as

$$
\bar{s}=\frac{1}{M N} \sum_{k=1}^{N} \sum_{n=1}^{M} \hat{s}_{k}(n)
$$

It is observed that if $\mathrm{p}$ is chosen to be greater than $\mathrm{L}+\mathrm{L}_{\mathrm{f}}$, the noise effect and the inter symbol interference produced by the fading channel impulse response and the low pass filter $\mathrm{f}(\mathrm{l})$ will be removed for $\mathrm{V}_{\mathrm{s}}^{\mathrm{N}}(\mathrm{pTs})$, moreover, even if $\mathrm{f}_{\mathrm{d}}=500 \mathrm{~Hz}, \mathrm{~L}_{f}$ is chosen to have $\mathrm{J}_{0}\left[\omega_{\mathrm{d}}\right.$ $\left.\left(\mathrm{L}+\mathrm{L}_{\mathrm{f}}\right) \mathrm{T}_{\mathrm{s}}\right] \cong 1[6]$.

\section{RESULTS AND DISCUSSION}


The following considerations are done on broad band wireless system data block size $M=32$ symbols and Symbol interval $T_{s}=0.25 \mu \mathrm{s}$, The receive filter $p_{R}(\tau)$ and transmit filter $p_{T}(\tau)$ are normalized square root raised cosine filter .Its roll-off factor is 0.3 . The wireless physical fading channel $g(t, \tau)$ is having 12 taps with a $T_{s}$ spacing between every two taps. The impulse response of $T_{s}$-spaced composite channel has inter-tap correlations. with $L=12$ taps. The cyclic prefix length is considered as $M_{c p}=L-1=11$. The data block number $N$ is considered as 2.A low-pass filter with $L_{f}=4$ taps is considered with $T_{s}$ spacing, generated frequency selective Rician, Rayleigh and Nakagami fading coefficients. Also BPSK modulated signal $x(n)$ is generated. Then the signal $\mathrm{x}(\mathrm{n})$ is partitioned into $\mathrm{N}$ blocks each of length $\mathrm{M}$ symbols. Then added cyclic prefix to the signal and obtained the received signal $y(n)$ At the receiver, the prefix is discarded and the mobile speed.is estimated by applying the algorithm.

\begin{tabular}{|c|c|c|c|c|c|}
\hline \multirow{7}{*}{$\begin{array}{l}\text { Table } 1 \\
\text { the } \\
\text { Doppler } \\
\text { different } \\
\text { Rayleigh }\end{array}$} & \multirow{2}{*}{$\begin{array}{c}\text { Actual Doppler } \\
(\mathbf{H z})\end{array}$} & \multicolumn{2}{|c|}{$\begin{array}{c}\text { Estimated } \\
\text { Doppler(Hz) }\end{array}$} & \multicolumn{2}{|c|}{$\begin{array}{c}\text { Estimated } \\
\text { Mobile Speed }(\mathrm{m} / \mathrm{s})\end{array}$} \\
\hline & & $\begin{array}{c}\mathrm{SNR}= \\
\text { 3.0998dB }\end{array}$ & $\begin{array}{l}\text { SNR= } \\
5.14 d B\end{array}$ & $\begin{array}{c}\text { SNR= } \\
\text { 3.0998dB }\end{array}$ & $\begin{array}{l}\text { SNR= } \\
5.14 d B\end{array}$ \\
\hline & 100 & 135.66 & 134.19 & 45.22 & 44.73 \\
\hline & 200 & 239.11 & 210.60 & 79.7 & 70.2 \\
\hline & 300 & 306.30 & 314.60 & 102.1 & 104.86 \\
\hline & 400 & 385.36 & 402.40 & 128.45 & 134.13 \\
\hline & 500 & 515.15 & 496.87 & 171.71 & 165.62 \\
\hline
\end{tabular}

Shows estimated values for SNRs for fading channel.i.e, 3.0998dB, $5.14 \mathrm{~dB}$

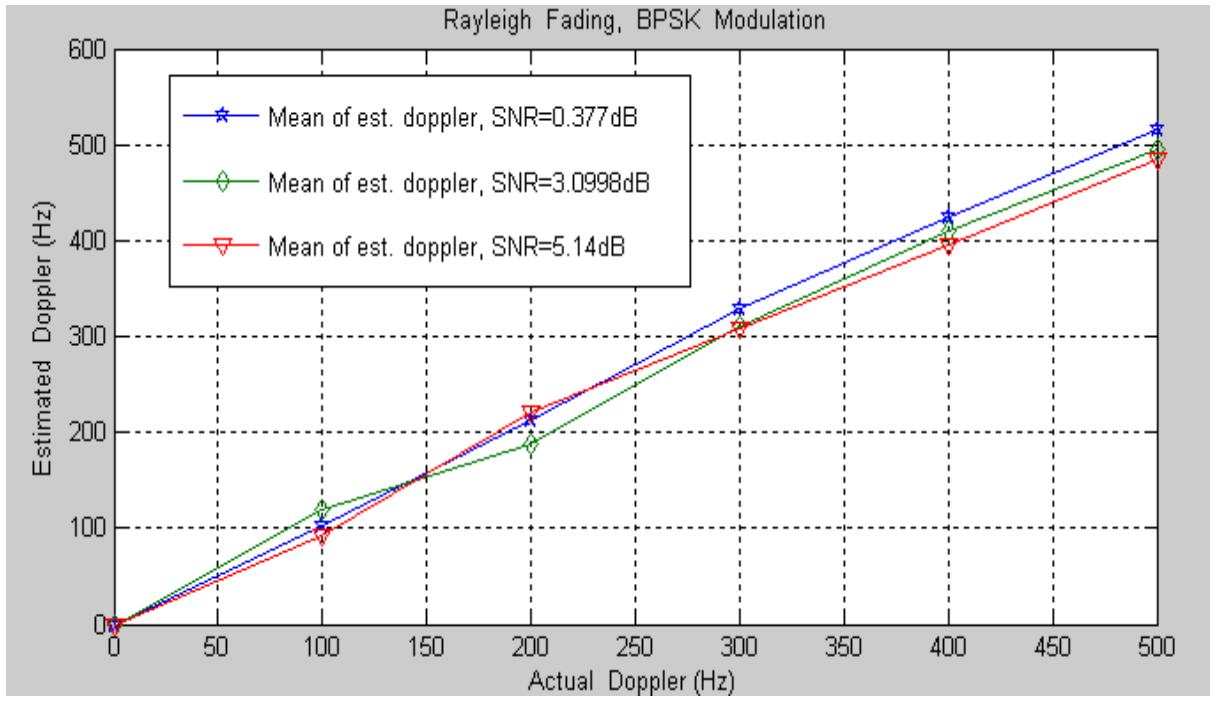

Figure 1 Estimation accuracy for Rayleigh fading channel with BPSK modulated signals.

Fig.1 shows the mean and standard deviation of the actual Doppler frequency versus the estimated Doppler frequency up to $500 \mathrm{~Hz}$ for different SNR values equal to $0.377 \mathrm{~dB}, 3.0998$ 
$\mathrm{dB}$ and $5.14 \mathrm{~dB}$. It is a frequency selective Rayleigh fading channel with BPSK modulated signals.

As seen a good estimation accuracy is provided by the proposed algorithm even if the SNR is as low as $0.377 \mathrm{~dB}$, and when SNR is $3.0998 \mathrm{~dB}$ or higher, the standard deviation of the estimated Doppler is found to be small.

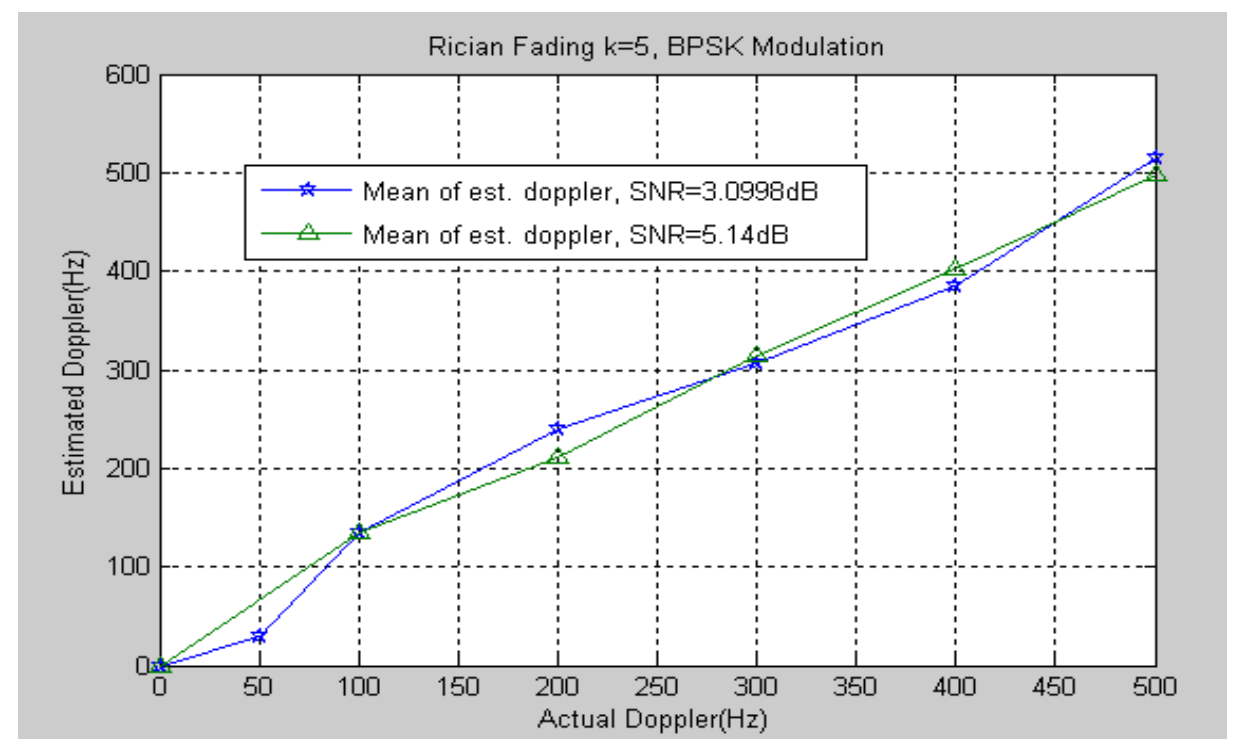

Figure 2 Estimation accuracy for Rician fading channel $(k=5)$ with BPSK modulated signals.

Fig. 2 shows the frequency selective Rician fading channels $(K=5)$ with BPSK modulated signals and the estimation accuracy of the algorithm. It is found that a reasonably good estimation accuracy is provided by the proposed estimation algorithm for BPSK modulated signals even if the SNR is as low as $3.0998 \mathrm{~dB}$, and when SNR is $5.14 \mathrm{~dB}$ or higher, the standard deviation of the estimated Doppler is fond to be small.

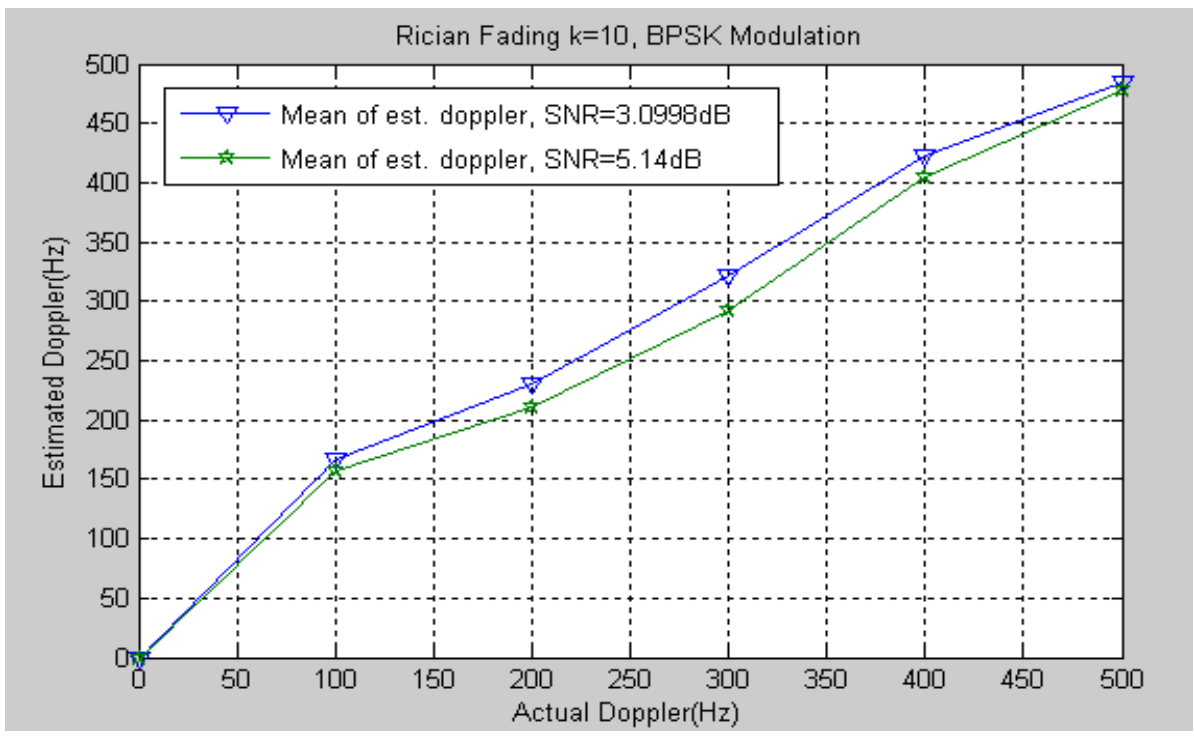

Figure 3 Estimation accuracy for Rician fading channel $(\mathrm{k}=10)$ with BPSK modulated signals.

Fig. 3 shows the frequency selective Rician fading channels $(K=10)$ with BPSK modulated signals and the estimation accuracy of the algorithm. 
It is found that a reasonably good estimation accuracy is provided by the proposed estimation algorithm for BPSK modulated signals even if the SNR is as low as $3.0998 \mathrm{~dB}$, and when SNR is $5.14 \mathrm{~dB}$ or higher, the standard deviation of the estimated Doppler is fond to be small. However, the deviation is found to be greater when compared to $k=5$ Rician fading channel.

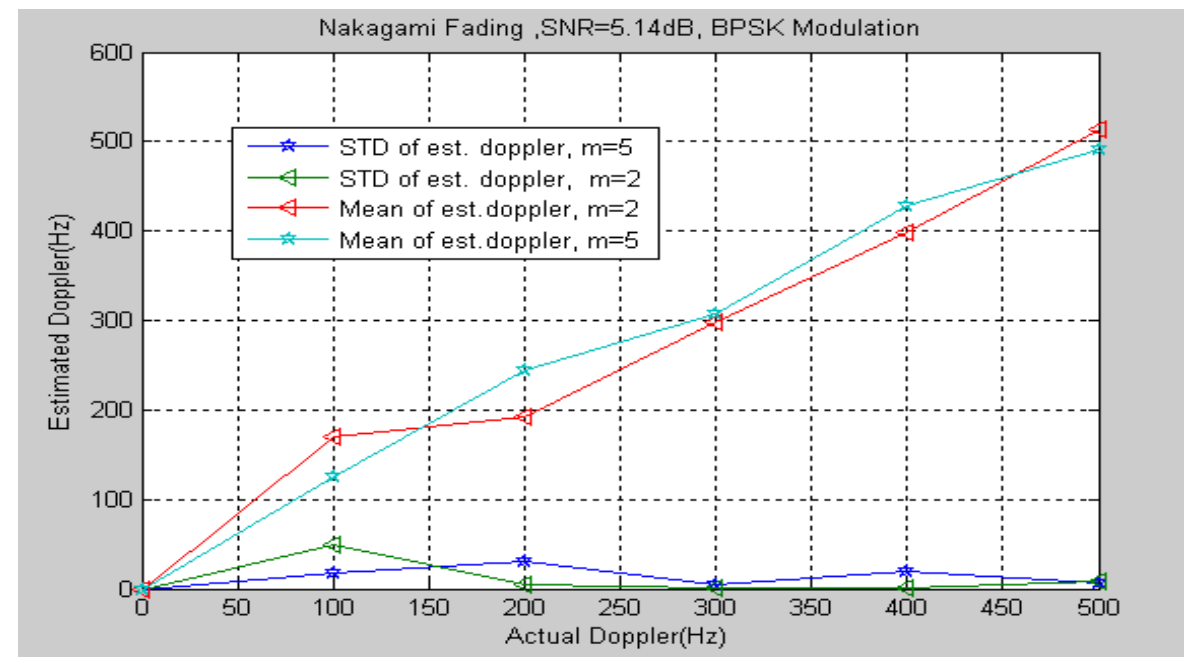

Figure 4 Estimation accuracy for Nakagami fading channels with BPSK modulated signals.

Fig. 4 shows the frequency selective Nakagami fading channels with BPSK modulated signals with $\mathrm{SNR}=5.14 \mathrm{~dB}$ and the estimation accuracy of the algorithm.

Here, the value of $m$ is considered as $m=2$ and $m=5$ for BPSK modulated signals. In case of Nakagami fading the deviation of estimated Doppler is higher for $\mathrm{m}=5$ compared to $\mathrm{m}=2$.

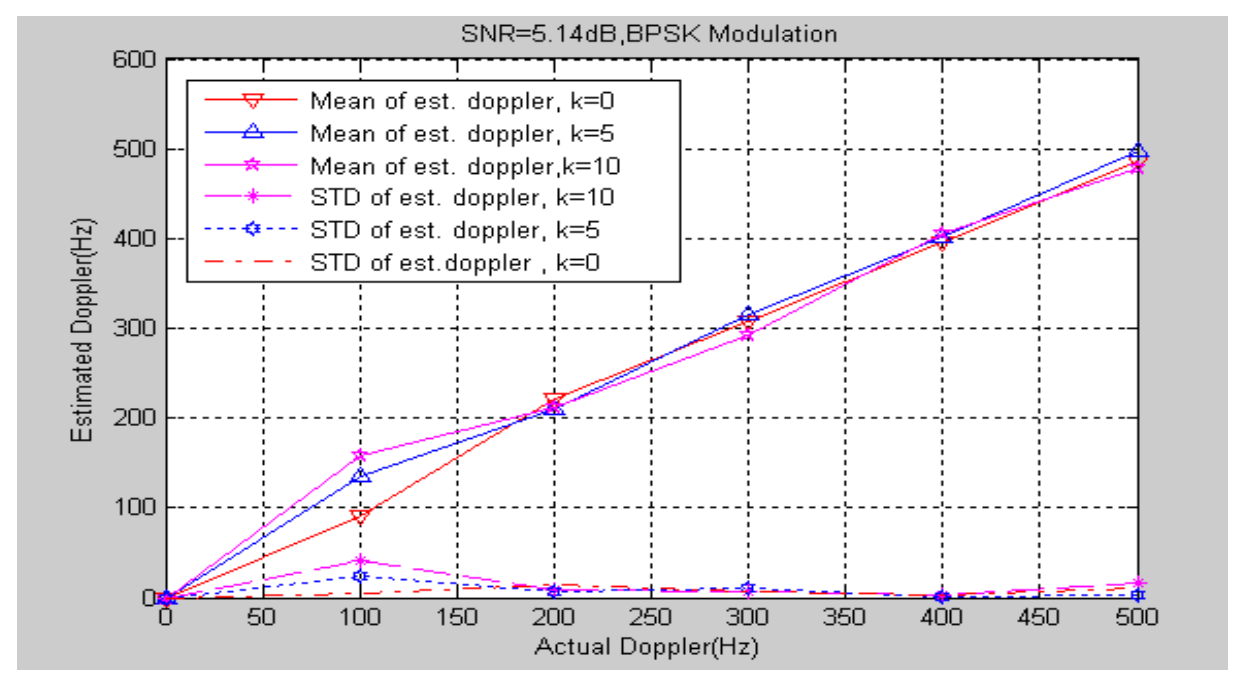

Figure 5 Standard deviation for Rician fading channels with BPSK modulated signal

Fig. 5. shows the plots of the standard deviation of Rician fading channels.

It is found that when the Rice factor $K$ increases the standard deviation of the estimated Doppler lean to be larger.For a particular SNR, at lower doppler frequencies the deviation is high for large value of $\mathrm{k}$, but deviation is negligible at higher doppler frequencies.

\section{CONCLUSION}


In this research it is found that the algorithm is insensitive to modulation schedules. The algorithm provides a better estimation results when the SNR is increased. A larger deviation on the estimation result is found when the Rice factor $K$ is increased. The algorithm shows a larger deviation on the estimation result when the Nakagami shape factor $m$ is increased. It is found that a better estimation results can be obtained if the slot number $N$ of data to be used for speed estimation is increased. But, the estimation accuracy decreases when the slot number $N$ and/or SNR is decreased.

\section{REFERENCES}

[1] Yahong Rosa Zheng, Chengshan Xiao, (2009) " Mobile Speed Estimation for Broadband Wireless Communications over Rician Fading Channels" Trans. Wireless Commun., vol. 8 .

[2] C. Xiao, J. Wu, S. Y. Leong, Y. R. Zheng, and K. B. Letaief, (2004) "A discrete-time model for triply selective MIMO Rayleigh fading channels," Trans. Wireless Commun., vol. 3, pp.1678- 1688.

[3] G. L. Stuber, (2001) Principles of Mobile Communications, 2nd Ed. Boston, MA: Kluwer.

[4] C. Xiao, K. D. Mann, and J. C. Olivier, (2001) "Mobile speed estimation for TDMAbased hierarchical cellular systems," IEEE Trans. Veh. Technol vol. 50 pp. 981- 991.

[5] J. Zhang, Q. Zhang, B. Li, X. Luo, and W. Zhu, (2006) "Energy-efficient routing in mobile adhoc networks: mobility-assisted case," Technol., vol. 55, pp. 369-379.

[6] M Haddad, DG Herculea. (2017) Online mobile user speed estimation: Performance and trade-off considerations2017 14th IEEE Annual Consumer Communications \& Networking Conference (CCNC) DOI: 10.1109/CCNC.2017.7983265

[7] K. Meena Anusha and Dr. Ch. Santhi Rani, (2016) Comparison of STBC System and STTC System Using Various Doppler Spectra, International Journal of Electronics and Communication Engineering and Technology, 7(6), pp. 01-13.

[8] Khyati Chopra, and K K Gupta, (2016) Hybrid LDPC and STBC Algorithms to Improve BER Reduction in OFDM Systems. International Journal of Electronics and Communication Engineering \& Technology, 7 (1), pp. 28 - 44

[9] D. Lalitha Kumari and Prof. M. N. Giri Prasad, (2017) A Review Paper on Performance Analysis of MIMO Based OFDMA System Under Fading Channel, International Journal of Electronics and Communication Engineering and Technology, 8(1), pp. 32-42.

[10] Joyanto Roychoudhary and Pushpendu Kanjilal, (2018) Phased Array Antenna Adaptive Beam forming for Space-Time Signal Processing Using Hybrid Genetic Algorithms with Mutation Operator Applied to Rayleigh Fading Channels. International Journal of Electronics and Communication Engineering and Technology, 9(4), pp.36-46 